\title{
Theoretical Study of the Electronic and Thermodynamic Properties of $\mathrm{Co}_{2} \mathrm{CrZ}(\mathrm{Z}=\mathrm{Ga}, \mathrm{Al})$
}

\author{
I. Asfour ${ }^{1, *}$, D. Rached ${ }^{1}$, Sébilleau Didier ${ }^{2}$, Ababou-Girard Soraya ${ }^{2}$ \\ ${ }^{1}$ Department of Physics, Faculty of Science, University Djillali Liabes, Algeria \\ ${ }^{2}$ Department of Nanosciences Materials, Institute of Physics of Rennes, University of Rennes 1, France
}

Copyright $\odot 2018$ by authors, all rights reserved. Authors agree that this article remains permanently open access under the terms of the Creative Commons Attribution License 4.0 International License

\begin{abstract}
The present work includes a first principle study of the electronic structure, elastic, magnetic and thermal properties of Co-based ternary full-Heusler alloys $\mathrm{Co}_{2} \mathrm{CrX}(\mathrm{x}=\mathrm{Al}, \mathrm{Ga})$. The lattice constant, bulk modulus, magnetic moment and density of states are studied using the full-potential linearized augmented plane wave method with the Generalized Gradient Approximation (GGA) as functional of exchange and correlation. This shows that the magnetic properties of the compound are dependent on electron concentration of main group element and all concentrations are magnetic in their equilibrium L2 structure. The calculations show that the alloys with all concentration are true half-metallic materials and exhibit $100 \%$ spin polarization at the Fermi level where it can be shifted within the energy-gap. In addition, the quasi-harmonic Debye model is applied to determine the thermal properties of the alloy.
\end{abstract}

Keywords DFT, FP-LAPW Method, Wien2k, Half-metalli, Heusler, Ferromagnetic

\section{Introduction}

With the development of spintronic devices in the recent years, materials with complete spin polarization at the Fermi level EF have attracted much attention because they are of great interest for scientific research and industrial applications [1-3] .An important class of materials which are at present under intense study are the so-called half-metals [4]. Heusler alloys are a very interesting class of compounds as they are known to display various appealing properties such as magneto resistance, thermoelectric and shape memory effect etc. They have been nicely summarized in a comprehensive review [5]. These materials are hybrids between metals and semiconductors or insulators, presenting metallic behavior for one spin band and semiconducting for the other, and thus overall they are either ferro- or ferrimagnets with perfect spin-polarization at the Fermi level [6], Groot and his collaborators in a pioneering paper published in 1983 predicted the existence of half-metallicity in the case of the intermetallic Heusler alloy NiMnSb[7].

The Heusler ternary alloys are determined by the generic formulas X2YZ or XYZ [8, 9], The -Heusler alloys (X2YZ) where $X$ and $Y$ are transition metals, and $Z$ is an element of group III, IV or V, in some cases, $\mathrm{Y}$ is replaced by either a rare earth element. The X2YZ Heusler compounds crystallize in the cubic L21 (AlCu2Mn-type) structure with the space group Fm-3m. In this structure, $\mathrm{X}, \mathrm{Y}$ and $\mathrm{Z}$ atoms are placed on the Wyckoff positions $8 \mathrm{c}(1 / 4,1 / 4,1 / 4), 4 \mathrm{a}(0$, $0,0)$ and $4 \mathrm{~b}(1 / 2,1 / 2,1 / 2)$, respectively. The cubic X2YZ compounds can also be found in the CuHg2Ti type structure. This formal XYXZ-type structure exhibits Td symmetry with the space group F-43m. In that structure the two $\mathrm{X}$ atoms occupy non-equivalent positions in contrast to the L21 structure. This structure is frequently observed when the nuclear charge of the $\mathrm{Y}$ element is larger than the one of the $\mathrm{X}$ element from the same period, that is $\mathrm{Z}(\mathrm{Y})>$ $\mathrm{Z}(\mathrm{X})$ for two $3 \mathrm{~d}$ transition metals. In this structure, $\mathrm{X}$ atoms occupy the nonequivalent $4 \mathrm{a}(0,0,0)$ and $4 \mathrm{c}(1 / 4,1 / 4,1 / 4)$ positions, while $\mathrm{Y}$ and $\mathrm{Z}$ atoms are located on $4 \mathrm{~b}(1 / 2,1 / 2$, $1 / 2)$ and $4 \mathrm{~d}(3 / 4,3 / 4,3 / 4)$ Wyckoff positions, respectively [10]. The two compounds $\mathrm{Co}_{2} \mathrm{CrAl}$ and $\mathrm{Co}_{2} \mathrm{CrGa}$ these are new innovative materials for many application spinotronique, these alloys have a special interest because of their relatively high Curie temperature. The two parents Heusler materials $\mathrm{Co}_{2} \mathrm{CrAl}$ and $\mathrm{Co}_{2} \mathrm{CrGa}$ proved to be very prospective materials for advanced thermoelectric applications such as in solid-state refrigeration and power generation. Heusler's materials are the subject of many studies. This type of material attracts the interest of chemists and physicists of the solid because of their remarkable physical and magnetic properties frequently encountered. Co-based Heusler alloys, which are considered promising candidates for commercial spintronic applications, in particular because of their high Curie 
temperatures.

\section{Calculation Methodology}

This study is performed using density functional theory DFT [11.12] calculations within the framework of full-potential linearized augmented plane-wave (FP-LAPW) method as implemented in WIEN2K package [13]. The generalized-gradient approximation (GGA) is used for the exchange correlation potential [9].The muffin-tin sphere radii RMT were chosen as equal to 2.1, 2.3, 1.7 and 2.2 a.u for the $\mathrm{Co}, \mathrm{Cr}, \mathrm{Ga}$ and $\mathrm{Al}$ atoms, respectively. The plane wave cut-off parameter is taken as $\mathrm{RMT}^{*} \mathrm{Kmax}=8.5$, The Brillouin zone sampling was performed according to the Monkhorst-Pack scheme and for k-space integration, a $(14 * 14 * 14)$ mesh was used resulting in $104 \mathrm{k}$ points of the irreducible part in the Brillouin zone [12].Study of thermodynamic properties was performed within the quasi-harmonic Debye model implemented in the Gibbs program [13]. The quasi-harmonic Debye model allows us to obtain all thermodynamics quantities from the calculated energy-volume points. Detailed descriptions of this procedure can be found in Refs. [13-17].

\section{Results and discussion}

\subsection{Structural Properties}

In the first step, we present the results for the geometrical structure of the two ternary Heusler alloys $\mathrm{Co}_{2} \mathrm{CrGa}$ and $\mathrm{Co}_{2} \mathrm{CrAl}$ as well as the lattice parameter and bulk modulus. To obtain the equilibrium lattice constant and determine the stable structure of these alloys, we perform structural optimizations on $\mathrm{Co}_{2} \mathrm{CrGa}$ and $\mathrm{Co}_{2} \mathrm{CrAl}$ alloys for nonmagnetic (NM), magnetic (M) and configurations for both $\mathrm{AlCu}_{2} \mathrm{Mn}$-type (L2 $2_{1}$ and $\mathrm{CuHg}_{2}$ Ti-type structures. It is clearly seen that these structures are more stable in the $\mathrm{AlCu}_{2} \mathrm{Mn}$-type $\left(\mathrm{L} 2_{1}\right)$ than in the $\mathrm{CuHg}_{2}$ Ti-type (see Fig. 1). The calculated total energies versus volume are fitted to the empirical Murnaghan's equation of state [18] to determine the ground state properties.

\subsection{Electronic Properties}

To elucidate the nature of the electronic band structure, we also calculated the total and partial densities of states, as shown in Figures 2 most transport properties are determined on the basis knowledge of the density of states. Generally, in the vicinity of the Fermi level, the bands are mainly due to orbital states d of $\mathrm{Cr}$ and the transition metals; this is justified by the half metallic character.

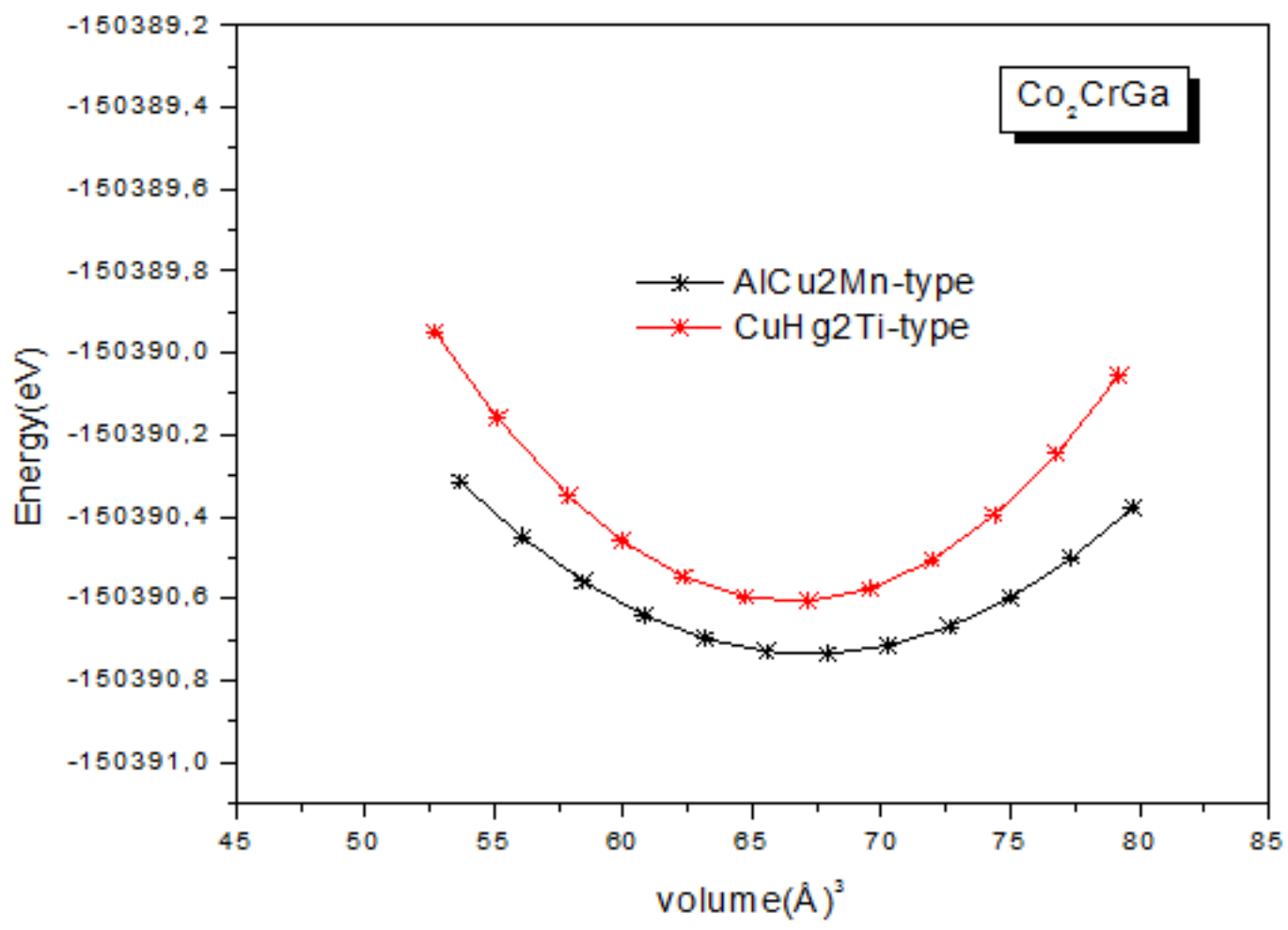




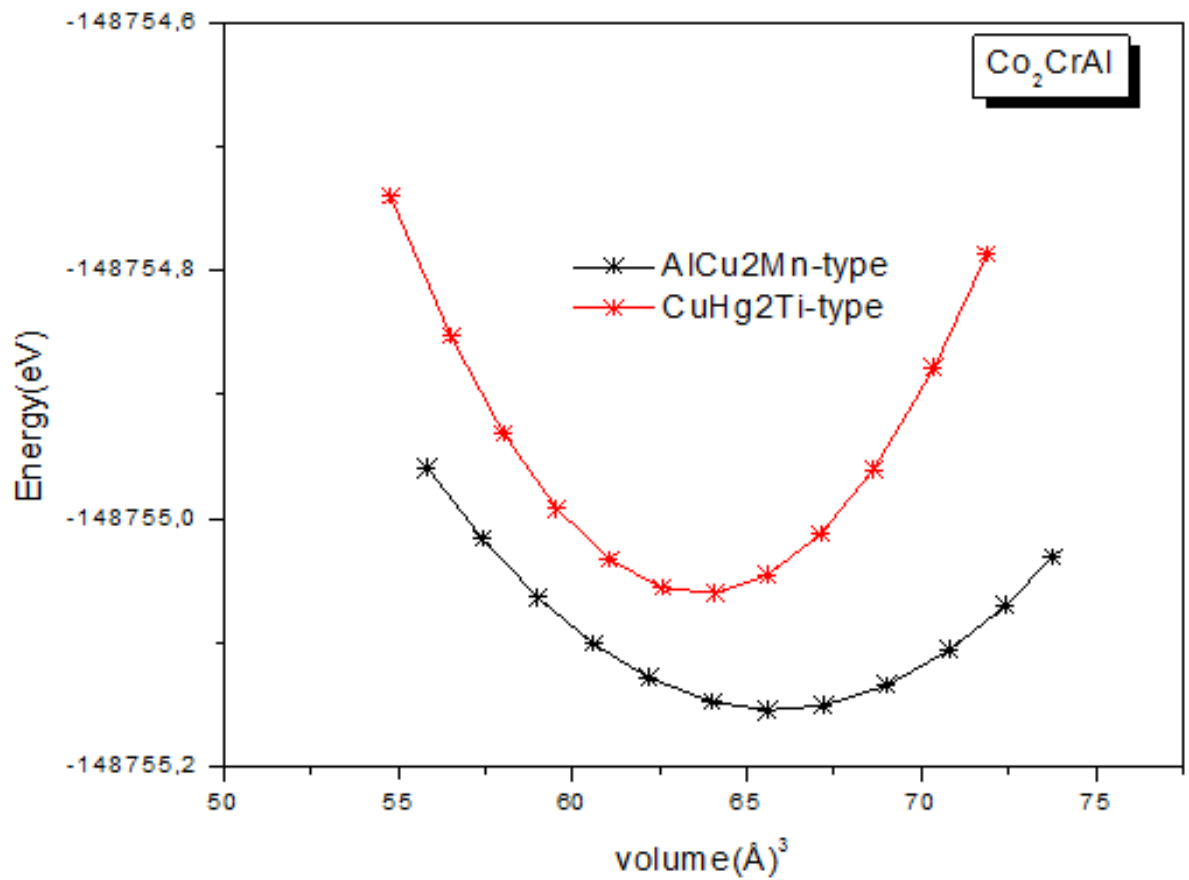

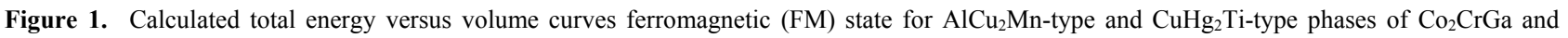
$\mathrm{Co}_{2} \mathrm{CrAl}$

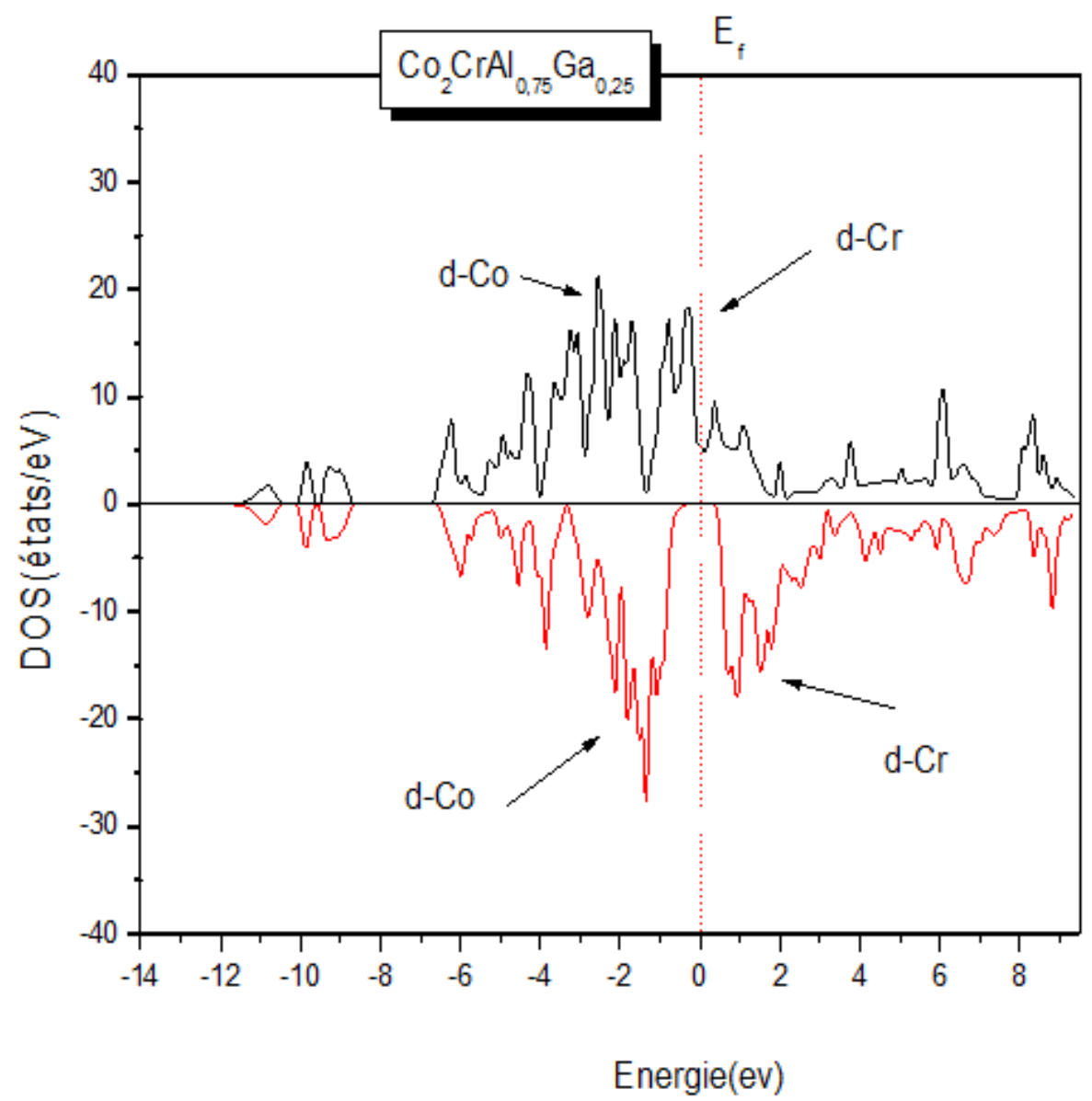



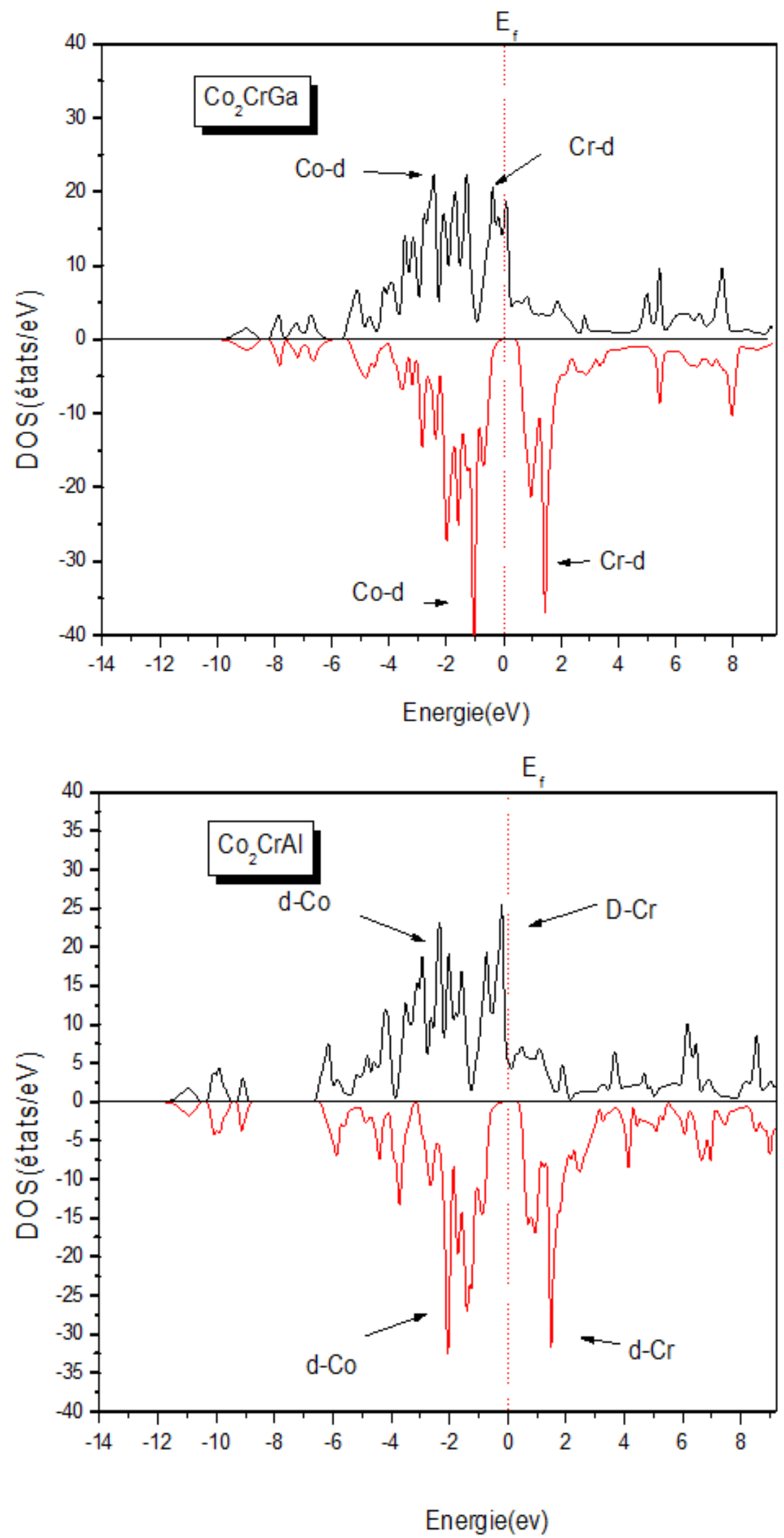

Figure 2. Total and partial density of states of the two ternary Heusler alloys $\mathrm{Co}_{2} \mathrm{CrGa}_{\text {and }} \mathrm{Co}_{2} \mathrm{CrAl}$. 
Densities of states confirming the metallic character for the majority of densities are presented in Figure 2. A lack of electronic states at the Fermi level of minority spins us closer to the semiconductor and semi-metallic character for both alloys. From figures 3, it is clear that there are three distinct regions in the spin-up state and in the spin-down state separated by gaps materials.

We notice an overlap valence and conduction bands to the state spin-up, while the existence of electronic states at
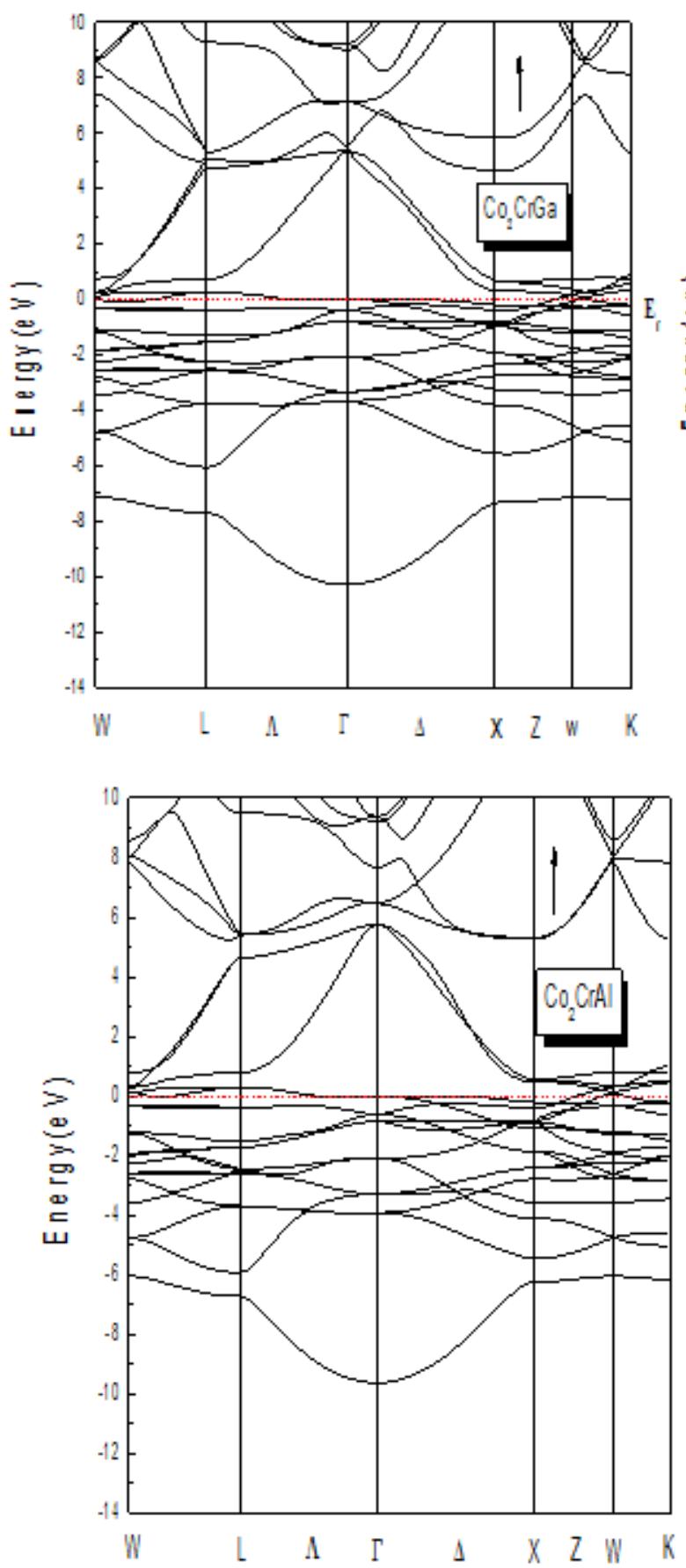

the Fermi level tells us about the nature of these metal alloys. The presence of electronic states is most obvious for the spin up, it is minimal for the spin down and shows the near character semiconductor, in this state we see a gap between the conduction and the valence band. It can be said that the compounds and their alloys are semiconductors in the state spin-dn. This means that the system has a half-metallic character. We notice a change in indirect gap for quaternary alloy materials studied
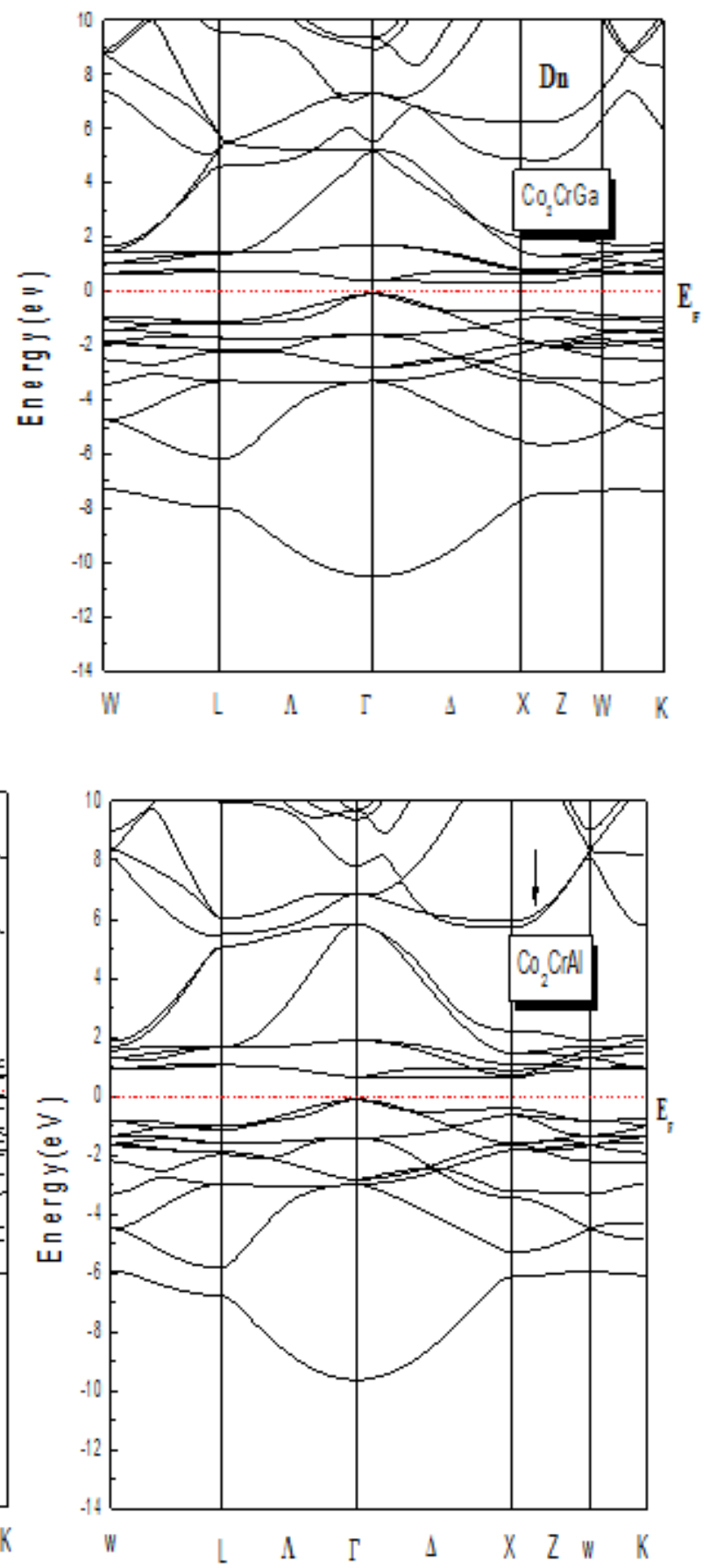

Figure.3. Calculated band structures of the two ternary Heusler alloy $\mathrm{Co}_{2} \mathrm{CrGa}$ and $\mathrm{Co}_{2} \mathrm{CrAl}$. 
We notice an overlap valence and conduction bands to the state spin-up, while the existence of electronic states at the Fermi level tells us about the nature of these metal alloys. The presence of electronic states is most obvious for the spin up against by it is minimal for the spin down and shows the near character semiconductor. Note that the maximum of the valence band and the minimum of the conduction band are located at the same point $\mathrm{X}$, so this is characteristic of direct gap. It can be said that the compounds and their alloys are semiconductors in the state spin-dn. This means that the system has a half-metallic character. Fig. 3 shows calculated band structures of the two ternary Heusler alloys $\mathrm{Co}_{2} \mathrm{CrGa}$ and $\mathrm{Co}_{2} \mathrm{CrAl}$.

The contribution to the determination of the structural (network parameter, incompressibility module), electronic (band structure, DOS density of states), mechanical, magnetic, thermal and thermodynamic properties of Heusler alloys.

\subsection{Magnetic Properties}

The term half-metallicity was introduced for the first time by Groot et al [19], focusing on the calculation of the half-Heusler alloy band structure: NiMnSb [20]. Conventional ferromagnetic materials have an electronic density of states

$\mathrm{N}(\mathrm{EF})$ ) at the Fermi level for the majority spin electrons (up: $N \uparrow(E F)$ ) and minority spin (down: $N \downarrow(E F)$ ). We can then define the polarization $\mathrm{P}$ in spin, which measures the spin asymmetry, by the expression [21]:

$$
P=\frac{N_{\downarrow}\left(E_{F}\right)-N_{\downarrow}\left(E_{F}\right)}{N_{\downarrow}\left(E_{F}\right)+N_{\downarrow}\left(E_{F}\right)}
$$

Half-metallic materials $(\mathrm{P}=1)$ show completely different conduction properties between minority and majority spins. They thus have a metal property for a spin direction (non-zero state density at the Fermi level) and a semiconducting or even insulating property for the other spin direction and consequently a spin polarization rate of $100 \%$

The magnetic moment per formula unit for the alloys of $3 \mathrm{~d}$ elements can be determined from the number of their valence electrons using Slater-Pauling (SP) rule [22].According to this rule, magnetic moment for the half metallic moment for the half metallic full-Heusler alloy is given by $\mathrm{Mt}=\mathrm{Zt}-24$, where $\mathrm{Mt}$ is the total magnetic moment per unit cell and $\mathrm{Zt}$ is the total number of valence electrons. The magnetic moment obtained by present calculations is $3.00010 \mu_{\mathrm{B}}$ and $3.0000 \mu_{\mathrm{B}}$ for $\mathrm{Co}_{2} \mathrm{CrAl}$ and $\mathrm{Co}_{2} \mathrm{CrGa}$ respectively which agrees well with values from SlaterPauling rule [23] as well as other calculations [24-26]. The energy band structure of a semi-metallic material has an asymmetry between the spin up states and spins down with a gap or a pseudo energy gap at the Fermi level. This gives rise to the polarizations of the conduction electrons at the
Fermi level which may reach $100 \%$.

The calculated total and local magnetic moment for constituent elements are given in Table1.

Table 1. Calculated total and partial magnetic moments (in $\left.\mu_{\mathrm{B}}\right)$ of the two ternary Heusler alloys $\mathrm{Co}_{2} \mathrm{CrGa}$ and $\mathrm{Co}_{2} \mathrm{CrAl}$

\begin{tabular}{|l|c|c|}
\hline $\begin{array}{l}\text { Magnetic } \\
\text { Moment }\left(\mu_{\mathrm{B}}\right)\end{array}$ & $\mathrm{Co}_{2} \mathrm{CrGa}$ & $\mathrm{Co}_{2} \mathrm{CrAl}$ \\
\hline $\mathrm{Co}$ & 0.76498 & 0.81226 \\
\hline $\mathrm{Cr}$ & 1.31271 & 1.43833 \\
\hline $\mathrm{M}$ & -0.04164 & -0.02216 \\
\hline Interstitial & 0.19912 & -0.04051 \\
\hline Total Moment $(\mu \mathrm{B})$ & 3.00010 & 3.00000 \\
\hline Other theoretical works & $3.0000^{\circ}$ & $3.0110^{\circ}$ \\
\hline Spin polarization $\%$ & 100 & 100 \\
\hline
\end{tabular}

${ }^{\circ}$ Ref.[27]

\subsection{Thermal Properties}

We can calculate the thermodynamic quantities for the Heusler alloy $\mathrm{Co}_{2} \mathrm{CrAl}_{1-\mathrm{x}} \mathrm{Ga}_{\mathrm{x}}$ through the Debye model quasi-harmonic implemented in the Gibbs program [28.29] in which the non-equilibrium Gibbs function $\mathrm{G}^{*}(\mathrm{~V}, \mathrm{P}, \mathrm{T})$.

Through the quasi-harmonic Debye model, one could compute the thermodynamic quantities of any temperatures and pressures of the ternary Heusler alloys $\mathrm{Co}_{2} \mathrm{CrGa}$ and $\mathrm{Co}_{2} \mathrm{CrAl}$ from the calculated $\mathrm{E}-\mathrm{V}$ data at $\mathrm{T}=0$ and $\mathrm{P}=0$. ( $\mathrm{T}$ being the temperature and $\mathrm{P}$ the pressure).

The relationship between the lattice parameter and the temperature at different pressures is shown in Figures 4-5 the lattice parameter increases to a very moderate with temperature. On the other hand, it is noted in Figures 6-7 that the relationship between the bulk modulus and the pressure is virtually linear. The bulk modulus increases with pressure and decreases with temperature.

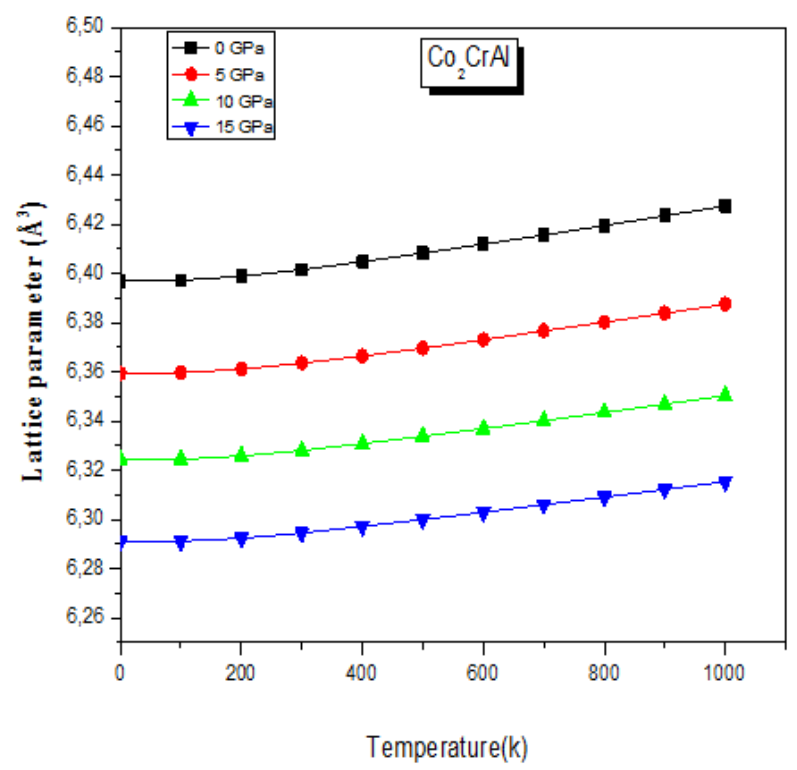

Figure 4. Variation of the lattice parameter as a function of temperature and pressure in $\mathrm{Co}_{2} \mathrm{CrAl}$ alloy. 


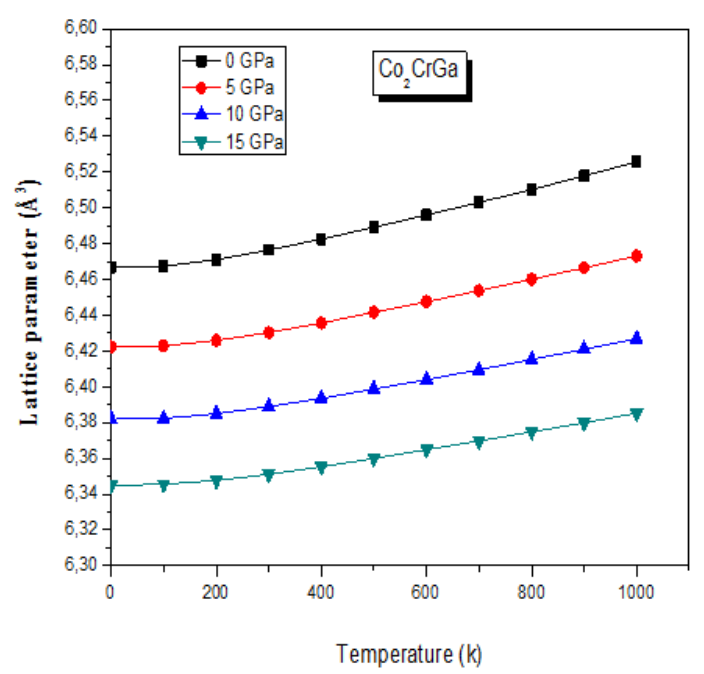

Figure 5. Variation of the lattice parameter as a function of temperature and pressure in $\mathrm{Co}_{2} \mathrm{CrGa}$ alloy.

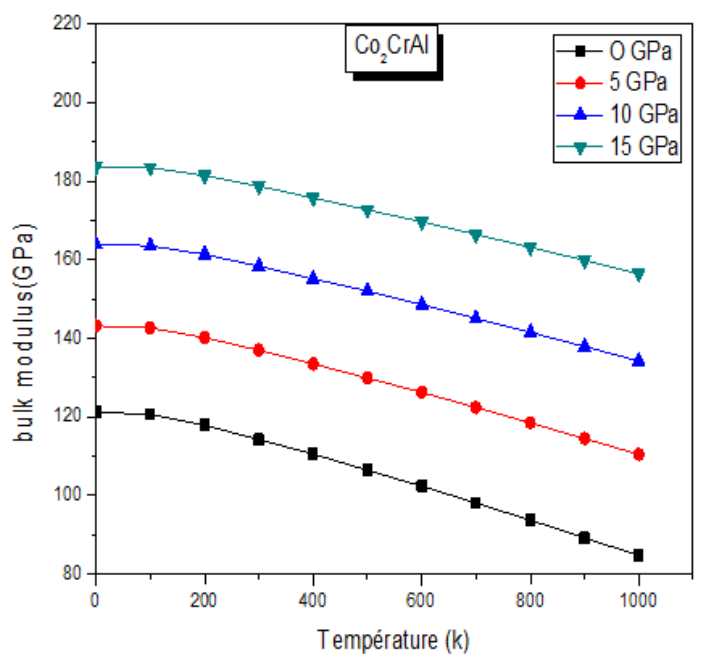

Figure 6. Variation of the bulk modulus as a function of temperature and pressure in $\mathrm{Co}_{2} \mathrm{CrAl}$ alloy.

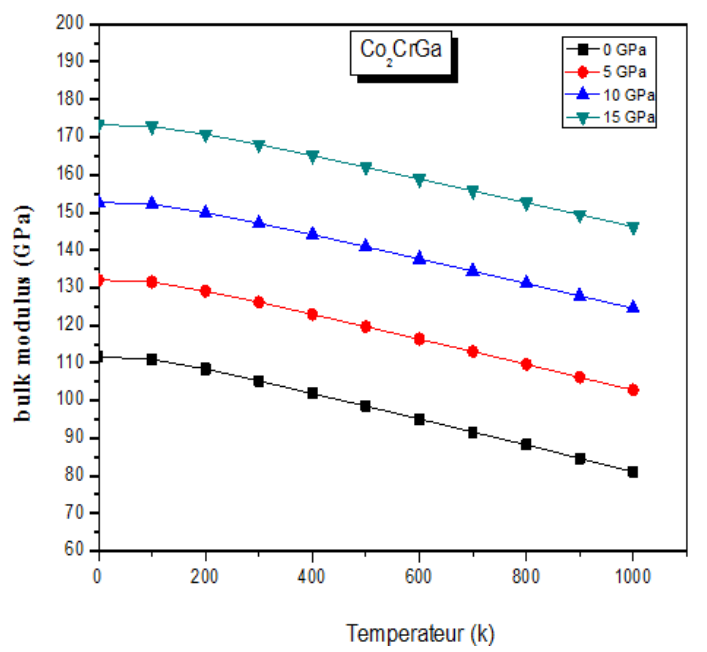

Figure 7. Variation of the bulk modulus as a function of temperature and pressure in $\mathrm{Co} 2 \mathrm{CrGa}$ alloy.

The investigation on the heat capacity of crystals is an old topic of the condensed matter physics with which illustrious names are associated. Knowledge of the heat capacity of a substance not only provides essential insight into its vibrational properties but is also mandatory for many applications. Two famous limiting cases are correctly predicted by the standard elastic continuum theory [30]. At high temperatures, the constant-volume heat capacity Cv tends to the Petit and Dulong limit [31], the Figures 8 and 9 show the variation of the heat capacity Cvcontant volume depending on the temperature for different pressures. This quantity indicates a strong increase up to $\sim 500 \mathrm{~K}$, which is due to anharmonic approximation Debye model. Values are purely predictive since we have no experimental data. However, at higher temperatures and at higher pressures, the effect of anharmonic $\mathrm{Cv}$ is deleted, and $\mathrm{Cv}$ tends towards the limit of Dulong-Pettit. $\quad(\mathrm{Cv} \quad(\mathrm{T}) \quad \cong$ 74.60J.mol-1.K-1-74.46J.mol-1.K-1, respectively $\mathrm{Co} 2 \mathrm{CrAl}$ and $\mathrm{Co} 2 \mathrm{CrGa}$ is shown in figure 8-9.

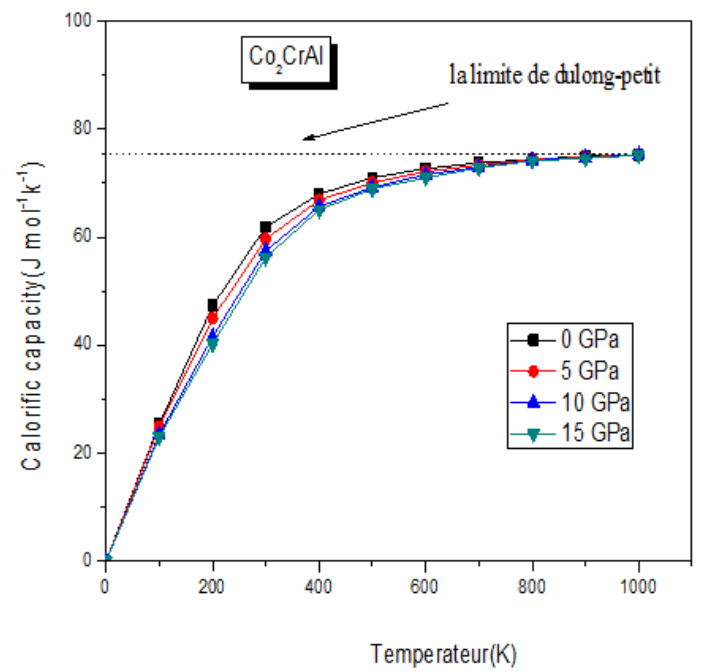

Figure.8. Variation of the heat capacity as a function of temperature and pressure in $\mathrm{Co}_{2} \mathrm{CrAl}$ alloy.

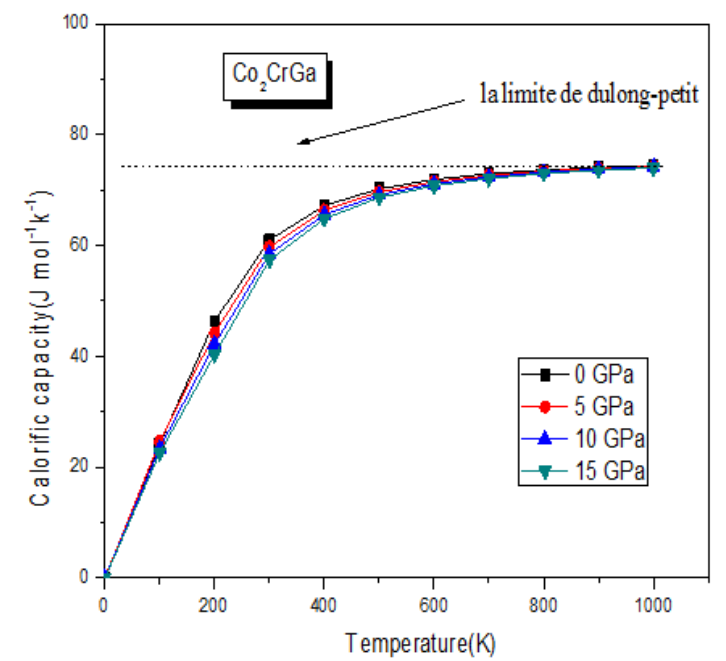

Figure 9. Variation of the heat capacity as a function of temperature and pressure in $\mathrm{Co}_{2} \mathrm{CrGa}$ alloy. 
In figures 10 and 11 , we present the variation of the Debye temperature $\theta \mathrm{D}$ as a function of temperature and pressure, respectively, one can observe that $\theta \mathrm{D}$ is nearly constant from 0 to $100 \mathrm{~K}$ and decreases linearly with increasing temperature from $\mathrm{T}>200 \mathrm{~K}$. It can be seen that the Debye temperature $\theta \mathrm{D}$ increases with pressure and decreases with temperature.

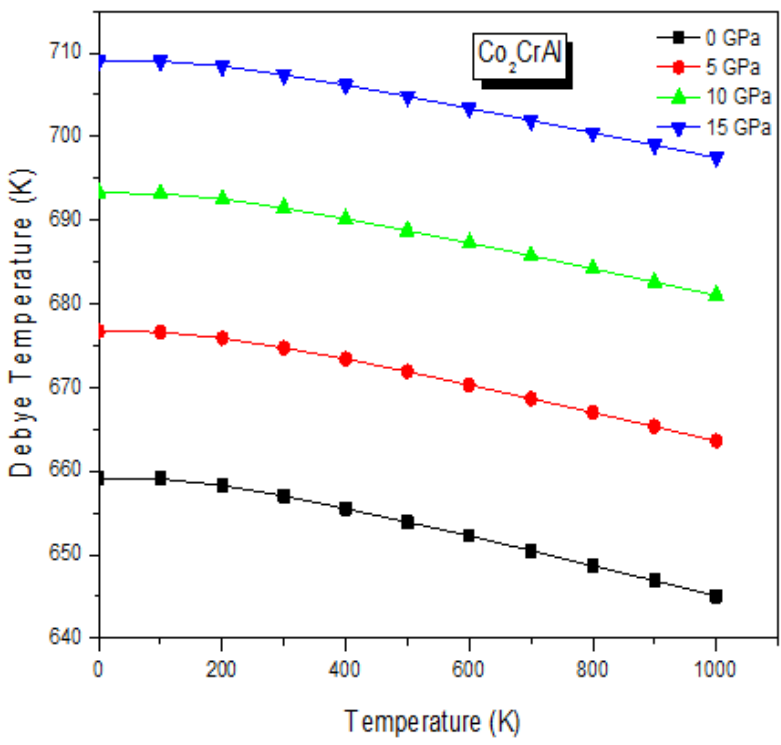

Figure 10. The Debye temperature $\theta_{D}$ as a function of temperature and pressure in $\mathrm{Co}_{2} \mathrm{CrAl}$ alloy.

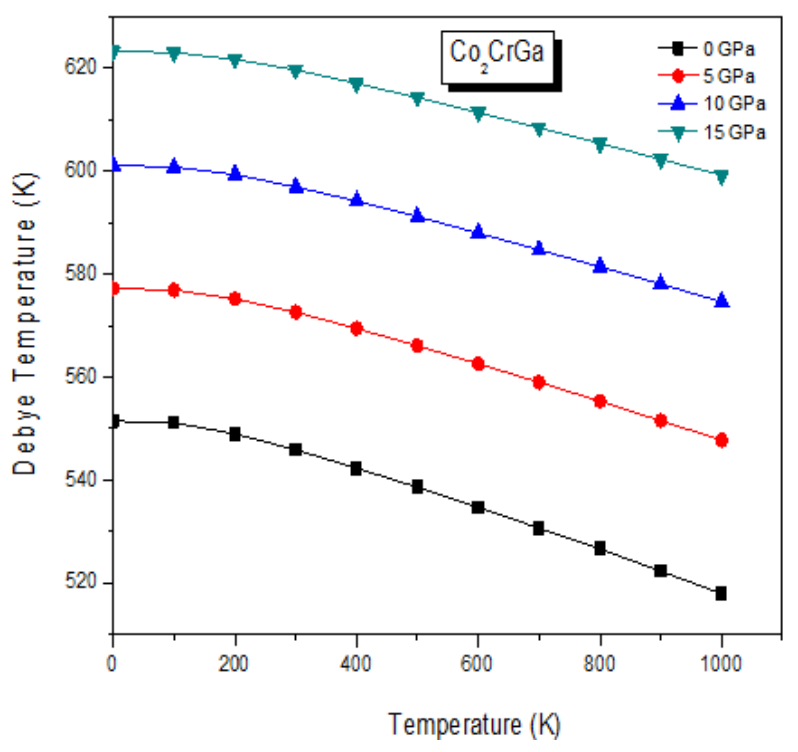

Figure 11. The Debye temperature $\theta_{D}$ as a function of temperature and pressure in $\mathrm{Co}_{2} \mathrm{CrGa}$ alloy.

The coefficient of thermal expansion $\alpha$ has been predicted Figur.12-13, He quickly believes in cube power of temperature then tends towards a limit.

We note, for a given pressure $\alpha$ increases with temperature when $\mathrm{T} \leqslant 300 \mathrm{~K}$ (at low temperature), especially at zero pressure, and tends gradually to increase linearly at higher temperatures. As the pressure increases, the variation of a with the temperature becomes smaller. For a given temperature, $\alpha$ decrease sharply with increasing pressure, and is very low at higher temperatures and higher pressures as well.

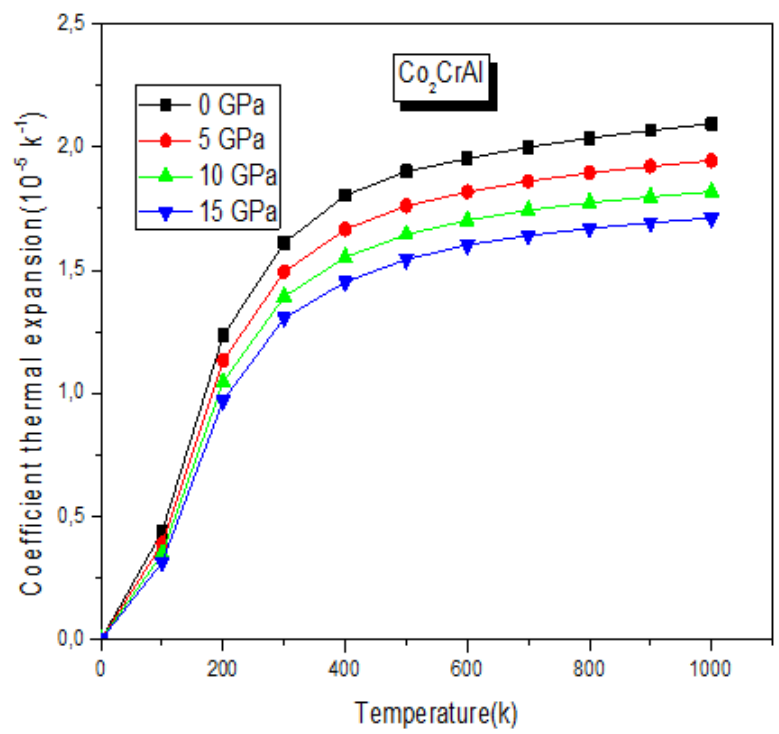

Figure 12. variation temperature coefficient as a function of temperature for different pressures for $\mathrm{Co}_{2} \mathrm{CrAl}$

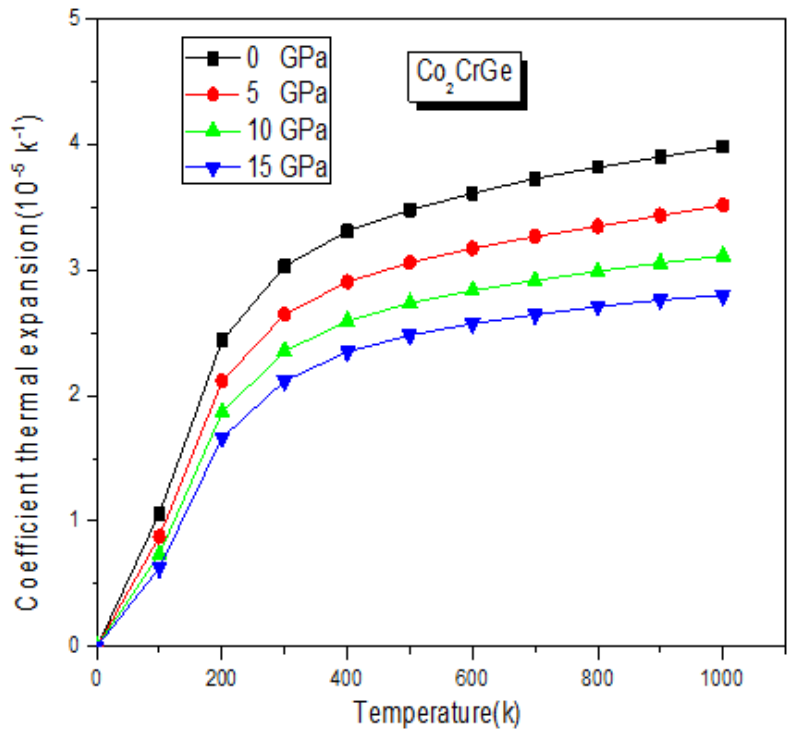

Figure 13. Variation temperature coefficient as a function of temperature for different pressures for $\mathrm{Co}_{2} \mathrm{CrGa}$

\section{Conclusions}

In this paper, by means of the first-principles calculations, we studied in detail the structural, elastic, electronic, magnetic, thermodynamic and thermal properties of the two ternary Heusler alloys $\mathrm{Co}_{2} \mathrm{CrAl}_{\mathrm{a}}$ and $\mathrm{Co}_{2} \mathrm{CrGa}$ at ambient as well as at elevated temperatures. With the linearized augmented plane wave method based on density functional theory and implemented in wien $2 \mathrm{k}$ 
code. For exchange correlation potential we have used the generalized gradient approximation (GGA) of Perdew et al. Our interest in this study was justified by the fact that the properties of these compounds are not available in the literature. The choice of compounds was warranted by the great deal of attention given to these Heusler alloys because of their large field of applications. The electronic band structures show a half- metallic- character. The quasi-harmonic Debye model is success-fully applied to determine the thermal properties at different temperatures and pressures. Finally, the results presented in this paper for the thermodynamic and thermal properties are predictions, and the experiments to prove them are welcomed.

\section{REFERENCES}

[1] R.A. de Groot, F.M. Mueller, P.G. van Engen, K.H.J. Buschow, Phys. Rev. Lett. 50 (1983) 2024.

[2] I. Zutic, J. Fabian, S. Das Sarma, Rev. Mod. Phys. 76 (2004) 323.

[3] C. Felser, G.H. Fecher, B. Balke, Angew. Chem. Int. Ed. 46 (2007) 668.

[4] I.Galanakis, P.H. Dederichs (Eds.), Half-Metallic Alloys: Fundamentals and Applications, in: Lecture Notes in Physics, vol. 676, Springer, Berlin,Heidelberg, 2005.

[5] T. Graf, C. Felser, S. Parkin, Prog. Solid State Chem. 39 (2011) 1-50

[6] I. Galanakis, Ph. Mavropoulos, P.H. Dederichs, J. Phys. D: Appl. Phys. 39(2006) 765.

[7] M. Parsons, J. Grandle, B. Dennis, K. Neumann, K Ziebeck, J. Magn. Magn.Mater.185 (1995) 140.

[8] E.P. Wohlfahrth, K.H.J. Bushow, Ferromagnetic Materials, vol. 4, Elsevier, Amsterdam, 1998.

[9] H.C. Kandpal, G.H. Fecher, C. Felser, J. Phys. D: Appl. Phys. 40 (2007) 1507.

[10] F. Heusler,W. Starck, E. Haupt, Verh DPG 5 (1903) 220.

[11] I. Asfour, H. Rached, S. Benalia, D. Rached, J. Alloy. Comp. 676 (2016) 440-451.

[12] M. Parsons, J. Grandle, B. Dennis, K. Neumann, K. Ziebeck, J. Magn. Magn. Mater. 185 (1995) 140.

[13] E.P. Wohlfahrth, K.H.J. Bushow, Ferromagnetic Materials, vol. 4, Elsevier, Amsterdam, 1998.

[14] H.C. Kandpal, G.H. Fecher, C. Felser, J. Phys. D: Appl. Phys. 40 (2007) 1507.

[15] I. Asfour, H. Rached, D. Rached, M. Caid, M. Labair, J. Alloy. Comp. 742 (2018) 736-750

[16] I. Asfour, D. Rached, Universal Journal of Mechanical Engineering 6(2) 21-37,2018.
[17] S.E. Kulkova, S.S. Kulkov, A.V. Subashiev, Computational Materials Science 36 (2006)249

[18] L. Heyne, T. Igarashi, T. Kanomata, K.U. Neumann, B. Ouladdiaf, K.R.A. Ziebeck, J Phys Condens Matter 17 (2005) 4991

[19] K. Hartjes, W. Jeitschko, J Alloys Compd 226 (1995) 81.

[20] M. Hehn, F. Montaigne, and A. Schuhl, "Magnétorésistance géante et électronique de spin." Techniques de l'Ingénieur l'expertise technique et scientifique de référence, 10 - Nov (2002).

[21] E. Francisco, M.A. Blanco, G. Sanjurjo, Phys. Rev. B 63 (2001) 094107.

[22] Blanco M A, Francisco E and Luana V 2004 Comput. Phys. Commun. 15857

[23] J.P. Perdew, S. Burke, M. Ernzerhof, Phys. Rev. Lett. 77 (1996) 3865

[24] J.F. Nye, Physical Properties of Crystals, Oxford University Press, 1985.

[25] F. Chu, Y. He, D.J. Thome, T.E. Mitchell, Scr. Metall. Mater. 33 (1995) 1295.

[26] D. P. RAI, A. SHANKAR, SANDEEP , M. P. GHIMIRE and R. K. THAPA India (2012)

[27] M. Florez, J.M. Recio, E. Francisco, M.A. Blanco, A. Martin Pendas, Phys. Rev. B

[28] E. Francisco, M.A. Blanco, G. Sanjurjo, Phys. Rev. B 63 (2001) 094107.

[29] J.P. Poirier, Introduction to the Physics of the Earth's Interior, Cambridge University Press, Oxford, 2000, p. 39.

[30] R. Hill, Proc. Phys. Soc. A 65 (1952) 349. 